\title{
UN ANÁLISIS SOCIOLÓGICO DE LAS POLÍTICAS DE TURISMO EN MÉXICO. TEPOZTLÁN, PUEBLO MÁGICO
}

\author{
MARIo Alberto VelÁZQUez García \\ marioalbertovelazquezg@gmail.com \\ El Colegio del Estado de Hidalgo \\ Helene Balslev Clausen \\ balslev@hum.aau.dk \\ Universidad de Aalborg
}

Este artículo propone analizar el turismo como una construcción social que posibilita la existencia de una serie de relaciones, valores, identidades económicas, culturales, políticas y relacionados con el desarrollo de esta actividad. La teoría "Construcción social de la población objetivo" es utilizada para analizar los factores que influyen en la selección, por parte de los Estados, de elementos generales para delinear sus políticas y sus acciones sobre distintos grupos sociales relacionados al turismo. Con ello, se analiza cómo el Estado transformó a Tepoztlán en un caso ejemplar no solo para el programa turístico federal Pueblos Mágicos, sino en la reconstrucción de la identidad nacional.

Palabras clave: turismo, estado, construcción social, Pueblos Mágicos, Tepoztlán.

\section{A SOCIOLOGICAL ANALYSIS OF TOURISM POLICIES IN MEXICO. TEPOZTLÁN, MAGICAL TOWN}

This article proposes to analyze tourism as a social construction that enables the existence of a series of relationships, values, economic, cultural, political identities, and those related to the development of this activity. The theory "Social construction of the target population" is used to analyze the factors that influence the selection, by the States, of general elements to outline their policies and actions on different social groups related to tourism. With this it is analyzed how the State transformed Tepoztlán into an exemplary case not only for the federal tourist program Pueblos Mágicos, but also in the reconstruction of the national identity.

Keywords: tourism, state, social construction, Pueblos Mágicos, Tepoztlán. 


\section{Introducción}

n este artículo sostendremos que el turismo es, socialmente hablando, más que un uso marginal del tiempo de descanso o una actividad exclusiva de la "burguesía". El turismo es una "conducta humana exploratoria" que utiliza manifestaciones culturales fuera de lo cotidiano para hacer que la vida sea significativa y digna de ser vivida (Graburn, 1989). En un plano macrosocial, el turismo es un "campus" de relaciones económicas, políticas y culturales más que movilidades, flujos de mercancías, modas, núcleos urbanos y reconfigura el uso de los espacios dentro de las grandes ciudades capitales del mundo (Urry, 2011; MacCannell, 1973).

El turismo es un fenómeno económico, de políticas públicas, pero también cultural y de configuración de la identidad para un estado nación. Ante ello, proponemos analizar la interacción entre los Estados con los agentes que participan en la definición y construcción de los lugares turísticos. En lo empírico retomaremos el caso de Tepoztlán, Morelos, una localidad incluida dentro del Programa Pueblos Mágicos (PPM).

El tema central que está detrás de este estudio es la relación que existe entre el turismo y el Estado. En específico cuáles son los mecanismos que estos últimos tienen a su disposición para generar una visión específica sobre los lugares, las actividades, las personas y la identidad nacional en las zonas turísticas. En otras palabras, analizaremos el cómo los Estados producen la construcción social de la realidad turística. La relación entre Estado y turismo resulta compleja, dado que implica: 1) intereses (individuales, grupales, políticos, económicos, etcétera), 2) dimensiones (económica, cultural, social, política), 3) lugares (playas, ciudades, bosques, lagos y otros), 4) procesos sociales (corta, mediana y larga duración) y 5) escalas de interconexión (entre individuos, comunidades, regiones, sociedades, naciones y global).

Para realizar este análisis elegimos uno de los puntos más directos de contacto entre el Estado y el turismo: las políticas públicas. Como lo define Cardozo (2006), las políticas públicas son un proceso social que implica un conjunto de decisiones, actuaciones y omisiones de los gobiernos relacionados a una serie de fenómenos administrativos, sociales y políticos particulares; todo esto ocurre dentro de un lugar y tiempo específicos buscando dar respuesta a una serie de problemas considerados como públicos por la sociedad y el Estado. Las políticas públicas son las herramientas centrales de los Estados en su búsqueda por generar una "realidad" social, es decir, regular, dirigir, transformar o prohibir comportamientos y fenómenos sociales a una población particular dentro de un territorio determinado. 
Reinsertar el análisis de las políticas públicas en una perspectiva social implica considerar que las decisiones de los Estados, en este caso aquellas sobre el turismo, se producen en un entorno donde existen actores con intereses y posturas distintas, incluso enfrentadas. Es decir, las políticas públicas se elaboran dentro de relaciones de poder que los Estados tienen con grupos y fenómenos múltiples. En este sentido, no consideramos al Estado como un ente homogéneo y coherente, sino como un campus de interacción entre grupos, intereses y visiones disímiles sobre lo público (Bourdieu, 2014).

\section{Metodología}

El material para esta investigación surge de un trabajo de campo de largo plazo realizado por los autores (Velázquez-García y Balslev) durante los años 2004-2005, 2011, 2012 y 2016 en Tepoztlán, Morelos. Durante estos años se realizaron estancias en esta localidad como parte de una red de investigación avocada a estudiar los municipios incluidos en el PPM.

La presencia durante distintos años permitió tener un conocimiento de primera mano sobre el desarrollo del turismo en Tepoztlán mediante entrevistas a profundidad y la observación participante (Fog y Kvale 1992). Se recolectaron materiales, se participó en visitas guidas sobre las historias, leyendas y tradiciones en el pueblo, observaciones y pequeñas charlas de las estancias para familiarizarse con el cómo y de qué manera los pobladores locales presentaban la ciudad, la historia y cómo promocionaban objetos, imágenes, medio ambiente y paisajes.

El análisis de Tepoztlán tiene como foco central la construcción social (estatal) de una localidad turística, en este sentido no se trata de una descripción histórica sobre la población. Por ello, aunque los elementos siguen un orden cronológico, el análisis mostrará como distintos elementos históricos han sido retomados por el estado, incluso a pesar de ser disímiles.

Todos los datos utilizados fueron verificados mediante el método de triangulación, lo que permite utilizar diferentes metodologías y con ello buscar que el tema sea dilucidado desde varios ángulos. Al utilizar la triangulación se reconoce que ningún procedimiento por sí mismo es capaz de analizar todo. 
Esta conjunción de métodos no busca extraer datos de las distintas fuentes y herramientas para verificar la verdad sino más bien intenta encontrar las conexiones que son centrales para la construcción de un análisis sociológico como el que aquí planteamos (Velázquez-García y Balslev, 2020). De esta forma, la triangulación no solo es la combinación de distintos datos, sino que constituye además un intento por relacionar diferentes tipos de información de manera tal que se evite caer en la trampa de que los datos produzcan un escenario de verdad aparentemente no problemático (Spradley, 1990).

El análisis de los datos empíricos crudos se mejoró considerablemente al incorporar las prácticas de los entrevistados dentro del marco sociocultural más amplio (Fog y Kvale, 1992). Otras fuentes de información son archivos históricos, libros y documentos históricos y la consulta de investigaciones sobre el turismo y los extranjeros en Tepoztlán (Velázquez-García, 2008). Los materiales secundarios permitieron complementar y contrastar las posturas sostenidas por esta investigación y en su caso completarlas (Fog y Kvale, 1992).

\section{Los significados sociales en la construcción de políticas públicas}

Como se mencionó anteriormente, las políticas públicas -en este caso de turismoson uno de los mecanismos estatales para promover que una determinada visión sobre las relaciones entre los grupos sociales, la definición de los problemas públicos y el uso de los recursos sean reconocidas por el resto de los grupos sociales como las indicadas, en este sentido las "correctas", adecuadas o las "apropiadas para dicha realidad". Este proceso no es un acto estatal unilateral, sino que se produce dentro de un entorno de interacciones donde existen fenómenos y actores que tienen capacidades para influir en ello.

A pesar de la importancia que tiene el conocer cómo y por qué son tomadas las decisiones estatales, estas preguntas son relegadas en los análisis sobre el desarrollo de las políticas públicas, especialmente en el caso del turismo. El conocer las ideas, los actores, las relaciones entre grupos y los elementos socioculturales permiten un mayor entendimiento del por qué el turismo comenzó a tomar relevancia dentro de los planes estatales. Esto resulta particularmente importante para los países de Latinoamérica, donde los trabajos de turismo generalmente se enfocan en temas 
administrativos, financieros, organizativos o económicos, sin cuestionar el por qué debe adoptarse el turismo como el nuevo modelo de crecimiento.

La definición de realidad de Berger y Luckman (1967, p. 11): "una cualidad propia de los fenómenos que reconocemos como independientes de nuestra propia volición", proporciona en el análisis sobre la elaboración de las políticas públicas una dualidad analítica.

En un primer momento, las políticas públicas toman como una realidad dada la presencia y las características socialmente atribuidas a un conjunto de actividades y sujetos sociales, a partir de lo cual se define su papel o destino de las políticas de gobierno. En segundo lugar, estas acciones estatales buscan generar una realidad transformada. Es decir, las políticas públicas no solo reconocen la presencia de ciertos grupos y sus formas de interrelación, sino que buscan alterar comportamientos e incluso, disminuir (o aumentar) la presencia o los efectos de determinados agentes sociales. En otras palabras, los Estados influyen en la transformación de la realidad mediante las políticas públicas y, con ello, en la forma en que un grupo o relación es percibida como real.

Para Berger y Luckman (1967) la realidad que percibimos contiene una serie de características, estas resultarán importantes dentro del campus estatal donde están situados los grupos, comportamientos y problemas que buscan ser el foco de atención de una política pública (Bourdieu, 2014). Las cinco propiedades de la realidad son: 1) intencionalidad, 2) intersubjetividad, 3) temporalidad, 4) orden y objetividad y 5) zonas no problemáticas (rutinas) y problemáticas. A continuación, explicaremos estas características.

\section{La mirada de lo real en la elaboración estatal de las políticas públicas}

En este apartado analizaremos los elementos planteados por Berger y Luckman (1967) sobre la percepción de la realidad en el caso de la construcción estatal de las políticas públicas, lo que permitirá analizar los diferentes grupos, motivaciones, imaginarios y otros elementos que intervienen en ese proceso.

El primer elemento es la intencionalidad. En el caso del Estado, esto constituye un ejercicio de poder mediante el cual busca intervenir sobre el campo de acción real o 
posible de los ciudadanos que habitan en su territorio. El poder puede ser definido como la posibilidad de ampliar o de restringir el campo de acción de los otros que se reconocen como actuantes y responsables, capaces de intervenir y, sobre todo, de responder (Foucault, 1991; Dean, 1999).

Una de las características centrales de los Estados modernos es su incesante búsqueda por conducir la conducta de todos los actores que se encuentran dentro de su territorio (Foucault, 2004). Las políticas públicas son los mecanismos concretos por medio de los cuales los Estados ejercen este control directo e indirecto sobre las conductas.

En el análisis de la intencionalidad estatal en las políticas públicas resultan centrales las capacidades (los recursos organizativos, monetarios, conocimientos, procedimientos legales), que tienen las estructuras estatales concretas para poder influir en la reconfiguración de un fenómeno específico como el turismo.

Al analizar la intencionalidad el problema de los recursos toma importancia pues su existencia o carencia afecta directamente la capacidad estatal para transformar el curso de acción de un grupo o los procesos sociales que se desarrollan dentro de un lugar determinado. En el caso de los Estados latinoamericanos, no ha sido hasta fechas relativamente recientes en que han desarrollado los mecanismos y contado con los medios necesarios para influir de una manera decisiva en el desarrollo del turismo dentro de sus propios territorios.

El segundo elemento referido, la intersubjetividad hace referencia a la multiplicidad de visiones, experiencias e intereses que pueden intervenir en el funcionamiento del Estado. En el caso de las políticas públicas, esta intersubjetividad se traduce en complejidad, es decir, la necesidad de considerar los distintos intereses de los actores involucrados, incluido el mismo Estado, para impulsar o imponer una serie de temas o trenes de acción.

En este sentido, la construcción social permite problematizar el diseño de políticas al reconocerlo como un fenómeno no-acabado, donde no existe una visión única sino, al contrario, diferentes visiones, intereses y perspectivas; el diseño de las políticas está determinado por el poder de los distintos grupos y la forma en que distintos sectores son percibidos y estereotipados dentro de una coyuntura social específica (Schneider e Ingram, 1993). 
En los diferentes ámbitos de gobierno los actores políticos y sociales buscan beneficiarse de los recursos disponibles. Para ello utilizarán un conjunto de estrategias de acción, entre otras formas de organizarse y participar. Con ello, se involucran en los espacios políticos y de política municipal, buscando alcanzar sus objetivos y metas por medio de la captación de recursos públicos. Al involucrase múltiples grupos se generan ambientes de tensión, situación que puede aliviarse cuando las autoridades municipales y los representantes de la sociedad local logren acuerdos; el diálogo, la negociación y la participación institucionalizada permiten una gestión municipal más eficiente (Sánchez, 2005).

El tercer elemento es la temporalidad: aquí y ahora. A diferencia de lo que proponen Berger y Luckman (1967) sobre la manera en que la realidad se construye para los sujetos, en el caso del Estado retomamos la propuesta de Cohen et al. (1972) respecto a la existencia de cuatro flujos temporales:

El primero es el flujo de problemas, que no se limitan a trenes monocausales, sino que son generados por personas dentro y fuera de la organización, su construcción obedece a complejas relaciones de temas económicos, sociales y culturales. El segundo es el flujo de soluciones donde generalmente el Estado espera la aparición de un problema en el cual pueda usar dicho flujo como respuesta, es decir, contrario a lo señalado en los modelos racionales, aquí se asume que las soluciones surgen al margen del cómo se responda o no a un problema particular. Así, por ejemplo, las computadoras no fueron creadas para solucionar un problema sobre nóminas en las empresas, pero permitieron solucionar este asunto (Cohen et al. 1972).

El tercero es el flujo de los participantes o actores involucrados. Este se caracteriza porque los individuos constantemente entran y salen de los procesos (de decisiones de política); su rotación depende de diferentes factores. Finalmente, las organizaciones se ven enfrentadas a un flujo de oportunidades donde se espera que, efectivamente, se tome alguna decisión.

En esta cadena de acontecimientos se unen los otros tres flujos con sus propias características para determinar el curso de la acción organizacional o grupal. Pero, por su misma naturaleza, las organizaciones no necesariamente tratan de cubrir objetivos claramente definidos o solucionar problemas específicos, muchas veces se busca actuar del mejor modo posible dadas las circunstancias y simplemente seguir funcionando. 
El cuarto elemento señalado por Berger y Luckman (1967) es que la realidad se presenta como ordenada y objetivada, es decir, contiene ya elementos designados que funcionan bajo pautas establecidas previamente. Sin embargo, es necesario cuestionar este supuesto, al menos para el caso de las políticas públicas.

El trabajo de March y Olsen (1989) mostró que uno de los problemas más evidentes al aplicar un modelo racional de toma de decisiones en la confección de las políticas públicas es el supuesto según el cual la realidad es única, objetiva y terminada. Ambos autores mostraron que un tomador de decisiones de política se enfrenta con una realidad difusa, es decir, cada organización realiza interpretaciones diferentes e incluso los miembros dentro de cada una refieren definiciones heterogéneas respecto a los hechos que las rodean.

En el caso del turismo significa que los intereses por los que un gobierno local o las diferentes agencias del gobierno federal impulsan un proyecto turístico pueden ser distintos. Como resultado, el sentido que cada grupo le otorga a la cadena de decisiones es ambiguo.

Las zonas no problemáticas y problemáticas hacen referencia a la serie de diferencias normativas, tanto las institucionalizadas en forma de leyes o normatividades como aquellas que tienen que ver con costumbres y prácticas culturales, que tendrán un efecto en el tipo de prácticas turísticas que serán permitidas en un lugar. En algunas regiones de Latinoamérica existen prácticas relacionadas al turismo que no serían permitidas, al menos no de una forma tan abierta, en otras regiones del mismo país como, por ejemplo, el consumo de bebidas alcohólicas en la vía pública, la tolerancia al consumo de drogas y la prostitución.

La percepción sobre las zonas no problemáticas (rutinas) y problemáticas resulta un problema inherente para la construcción de las políticas públicas. En ello interviene tanto la percepción de aquellos relacionados directamente, como también los imaginarios y prácticas de la sociedad en general (Hiernaux, 1997).

\section{El Programa Pueblos Mágicos}

El PPM es una política federal que comenzó a operar en el 2001, con la incorporación de 30 localidades en todo el país. Para el año 2021 estaban contempladas 121 localidades distribuidas en todos los estados de México. 
El Programa Pueblos Mágicos contribuye a revalorar a un conjunto de poblaciones del país que siempre han estado en el imaginario colectivo de la nación en su conjunto y que representan alternativas frescas y diferentes para los visitantes nacionales y extranjeros... Un Pueblo Mágico es una localidad que tiene atributos simbólicos, leyendas, historia, hechos trascendentes, cotidianidad, en fin, magia que te emanan en cada una de sus manifestaciones socio-culturales, y que significan hoy día una gran oportunidad para el aprovechamiento turístico. (Sectur, 2020).

El PPM fue diseñado para promover el turismo cultural independientemente de la especialización turística de cada estado, buscando impulsar la imagen urbana de las ciudades que tengan bienes patrimoniales materiales o inmateriales. Su objetivo general es "en un corto y mediano plazo, las localidades que reciben los beneficios del nombramiento de Pueblos Mágicos (PM) puedan fortalecer la infraestructura, la calidad de los servicios, la diversificación de sus productos turísticos, la creación y modernización de herramientas comerciales, acciones que contribuirán a detonar el crecimiento del mercado interno" (Velázquez-García y Lara, 2017).

En un plano general, podemos decir que el PPM tuvo resultados ambiguos: fortaleció la infraestructura turística (hoteles y restaurantes) en las localidades que participaron en un promedio de $148 \%$ y hubo un incremento en promedio de $234 \%$ en los recursos municipales (Velázquez-García y Lara, 2017).

Sin embargo, el programa presenta una serie de problemáticas: 1) la falta de coherencia entre las medidas tomadas y las políticas generales sobre turismo, 2) la poca cooperación entre las instituciones del programa, municipios, estados y federación en materia turística, 3) los gobiernos estatales y municipales no realizan una inversión complementaria, de forma que los recursos federales son insuficientes para consolidar una localidad como turística y 4) existían problemas en la operación de los comités PM.

Trabajos como el de Millán y Cota (2021) muestran cómo en algunos casos, por ejemplo, en las localidades del Estado de México que participan en el PPM, se registra una disminución de la pobreza. No obstante, todavía es necesario determinar hasta qué punto la variación en la pobreza puede ser atribuida al funcionamiento del PPM (Millán y Cota, 2021). En este trabajo se analizará el efecto que tiene una política turística en disminuir la pobreza en una localidad específica. 


\section{Tepoztlán: de la periferia al centro de la definición de México}

Tepoztlán es un municipio del Estado de Morelos que al norte limita con la Ciudad de México (delegación Milpa Alta) al oeste con los municipios de Huitzilac y Cuernavaca, al sur con Jiutepec y Yautepec, al este con Tlayacapan y Tlalnepantla. Esta localidad reingresó al PPM en el 2010. Para la Secretaría de Turismo (Sectur) los principales atractivos son: Pirámide del Tepozteco, Templo y exconvento de la Natividad, Museo Carlos Pellicer, Cruz de Axitla, Baño de Temazcal y Parque Nacional Tepozteco. La descripción sobre el lugar busca conjuntar los atractivos prehispánicos y contemporáneos:

Bendecido por una geografía que quita el aliento y un no sé qué que no deja de seducir a los visitantes, el pueblo resguardado por el Cerro del Tepozteco es tierra de misticismo, artesanías, clima inmejorable y relajación, pero sobre todo de buena vibra y energía que revitaliza. (Sectur, 2019).

El momento en que los primeros pobladores se asentaron en la zona que ocupa Tepoztlán no es claro, pero hay estimaciones de que ya existían asentamientos humanos entre 947 y 1156 de nuestra era. La zona ha sido repoblada por diversos grupos indígenas y ha sido objeto de guerras por su control. Moctezuma I tomó el control de este lugar en 1433. En 1523 Hernán Cortés inició la conquista de esta región mediante el establecimiento de una hacienda azucarera. En 1530 llegaron los Dominicos y el 8 de septiembre de 1538 se produce el bautizo del rey Tepoztécatl (Concheiro, 1998).

Tepoztlán fue una de las muchas comunidades indígenas que sufrió las consecuencias de la Revolución Mexicana; la población local disminuyó, la economía agrícola fue parcialmente abandonada y no existían instituciones de salud o educación permanentes. En estas condiciones sociales, Robert Redfield visitó Tepoztlán a fines de la segunda década del siglo XX. Como resultado de este trabajo de campo publicó en 1930 el libro Tepoztlán, a mexican village: a study of folk life.

Redfield escogió a Tepoztlán como el ejemplo ideal de una sociedad campesina. El antropólogo norteamericano buscaba demostrar que las pequeñas comunidades tienen una serie de rituales e instituciones que integran a sus miembros y les permiten resolver sus conflictos. Para él, este pueblo era un claro ejemplo de su idea de folk: una comunidad pacífica viviendo un ritmo de vida que les permitía a sus habitantes balancear los momentos de tensión y de calma (Redfield, 1953; 1955). 
La visión de Redfield (1930) resultaría fundamental para colocar a esta comunidad dentro del flujo de soluciones del gobierno respecto al desarrollo de turismo en pequeñas comunidades mediante el PPM: Tepoztlán reunía las condiciones buscadas (o imaginadas) para resaltar "lo mexicano": la tranquilidad y armonía de los pequeños pueblos.

La presencia de Redfield en Tepoztlán resultaría fundamental para esta comunidad. Primero, los escritos del antropólogo norteamericano (quien gozaba ya de un amplio reconocimiento internacional) iniciaron un proceso de revalorización por parte del Estado mexicano sobre la realidad objetiva y ordenada que representaban las prácticas y rituales indígenas, pasando de un modelo de atraso a ejemplo de armonía y de vida colectiva (Oseguera, 2008).

El número de personas interesadas en conocer este lugar comenzó a incrementarse (Velázquez-García y Balslev, 2012). Un ejemplo es Stuart Chase, quien publicó el libro Mexico: a study of two americas (1931). Chase visitó Tepoztlán para compararla con una comunidad norteamericana. En 1958 se filmaron escenas de exteriores de la película The Magnificent Seven (Los siete magníficos) en este pueblo.

La narrativa del Estado mexicano y del trabajo antropológico de Redfield coincidían en que Tepoztlán encarnaba la visión ideal del campesino mexicano: habitantes de pequeñas comunidades, guardianes de valores, ideales, tradiciones y dispuestos a pelear por sus derechos, pero diferían sustancialmente sobre el futuro de estos lugares: donde Redfield veía soluciones a las problemáticas de los procesos de urbanización en las ciudades contemporáneas, el Estado mexicano veía atraso y el reflejo de un México que debía dejarse atrás.

En otras palabras, comenzó a construirse una intersubjetividad y un conjunto de problemas y soluciones diferenciados respecto a Tepoztlán. Al mismo tiempo, este pueblo comenzó a institucionalizarse en un lugar significativo de la esencia de México (Lomnitz-Adler, 1995).

En 1943 Oscar Lewis viajó a Tepoztlán, describiendo una comunidad muy diferente a la vista por Redfield: mientras que este último encontró una sociedad estable y unida, Lewis vio un pueblo con serios conflictos. Por ende, su trabajo representaba una crítica a su antecesor, pues consideraba a Tepoztlán una sociedad muy lejos de ser homogénea, más bien caracterizada por sus problemas de integración y funcionando por medio de instituciones egoístas (selfish institutions), con falta de 
cooperación entre sus miembros y con un resentimiento generalizado hacia otras comunidades. Los tepoztecos, en la visión de Lewis, eran individuos llenos de miedos, envidia y desconfianza en sus relaciones interpersonales. En lugar del folk de Redfield, el pueblo debía ser caracterizado por su conflicto interno (Lewis, 1968; Lara, 2004).

Las posiciones diametralmente diferentes entre Redfield y Lewis generaron un debate dentro de la antropología norteamericana (Redfield, 1953 y 1955; Lewis, 1959). Esta polémica resulta ilustrativa en nuestro intento de entender la construcción de la población objetivo en México, dado que ambas posturas ilustran tanto la atracción que ejerce este tipo de comunidades campesinas mexicanas entre los visitantes norteamericanos, como los flujos temporales dentro del aquíy ahora del gobierno mexicano frente a una comunidad indígena- campesina.

La primera postura (Redfield) continúa la tradición occidental sobre las utopías, donde los grupos sociales no "contaminados" por la modernidad funcionan armónica y solidariamente (Velázquez-García, 2009b). Es decir, las comunidades pueden ser un flujo de soluciones turísticas para el gobierno, en este caso, para aquellos visitantes que buscan comportamientos y valores inexistentes en las ciudades, como la tranquilidad y la vida "simple" (Balslev, 2008).

La segunda postura (Lewis, 1951) es parte de una crítica a las consecuencias que tiene la interacción entre las sociedades tradicionales y la modernización bajo situaciones de desigualdad de recursos. En esta perspectiva, Tepoztlán es colocada dentro del flujo de problemas: la desintegración de comunidades que pierden sus valores sin adaptar sus prácticas a las sociedades modernas y están a espera que el gobierno solucione sus problemáticas (Redclift, 1986).

A finales de la década de los 50 y principios de los 60, ya existía un grupo de extranjeros viviendo en Tepoztlán (Lewis, 1951). Los foráneos no solo están compuestos por extranjeros sino por una importante cantidad de personas que procedían de la Ciudad de México. Estos últimos se caracterizaban por tener actividades laborales relacionadas a la cultura o la investigación (Lewis, 1968).

La presencia de estos dos grupos será fundamental en la construcción de Tepoztlán como un destino turístico y en la conformación de los grupos principales de la política del turismo; tanto los extranjeros como los dueños de casas son definidos como los beneficiarios del desarrollo turístico, no los habitantes locales. El crecimiento en 
la población de Tepoztlán es un fenómeno relacionado con el aumento de las actividades turísticas y de segundas residencias. En 1940 había 6 mil 034 habitantes, en 1960 llegó a 8 mil 265, en 1980 creció a 19 mil 122, en el año 2000 a 32 mil 921 y en el 2010 llegó a 41 mil 629 (Instituto Nacional de Estadística y Geografía, INEGI, 2015).

\section{El turismo New Age en Tepoztlán}

Los antropólogos Robert Redfield y Oscar Lewis no son los únicos extranjeros que han tenido una influencia decisiva en la construcción social que se ha hecho sobre Tepoztlán. Otro personaje fundamental es Daniel Ruzo (2012), quien publicó en 1978 un libro titulado El valle sagrado de Tepoztlán. Los templos Atlantes de México. El argumento central en este libro es que Tepoztlán constituye un centro magnético de la tierra y que en sus cerros existen restos de una civilización antigua relacionada con la Atlántida.

Desde el año de 1997 diversas personas locales y extranjeras que habitaban en Tepoztlán comenzaron a reportar la presencia de ovnis, principalmente ubicados sobre los cerros del Tepozteco (Azteca América, 2011; Diario de Morelos, 2020; Animal. $m x, 2020)$. El continuo reporte de avistamientos ha convertido a Tepoztlán en uno de los cinco lugares en Latinoamérica donde más turistas acuden buscando ovnis ${ }^{1}$.

Esta condición particular de Tepoztlán como zona "redescubierta" por Ruzo (2012) con un poder espiritual particular comenzó a ser retomada por diversos grupos que, desde la década de los 70 mezclaban prácticas orientales de yoga, meditación y elementos de las culturas prehispánicas mexicanas. Tepoztlán fue convirtiéndose en un punto de reunión para estas creencias de la llamada "nueva era", en parte, gracias a las ideas de Ruzo (2012). Esto generaría una nueva transformación en la temporalidad del aquíy ahora de la política de turismo en Tepoztlán, pues el "redescubrimiento de la espiritualidad" de la zona generó un nuevo grupo de negocios y visitantes. Esto reafirmo la "vocación" turística de la localidad.

En la actualidad, Tepoztlán es un centro de turismo "espiritual". En esta localidad existen 25 negocios donde se ofrecen clases de yoga y meditación. Entre estos establecimientos es posible observar diferencias notables; algunos son pequeños e im-

1 Los otros lugares son: Chilca (Perú), Lajas (Puerto Rico), Capilla del Monte (Argentina) y San Clemente (Chile) (González, 2012). 
provisados, con instructores no certificados; otros forman parte de lujosos centros de retiro y hospedaje con personal capacitado a nivel internacional.

En el año 2013, se organizó en esta ciudad el Congreso Nacional de Yoga. Esto se relaciona con un tercer flujo, en este caso, el de los involucrados en la toma de decisiones. En Tepoztlán existen establecimientos que podemos llamar "New Age": temazcales $^{2}$ (al menos 17 establecimientos), masajes (prehispánicos y orientales) y venta de piedras "curativas".

Los hoteles en Tepoztlán, particularmente aquellos exclusivos o boutique, incluyen yoga, temazcal y masajes como parte de sus servicios. También existen diversos locales y personas que ofrecen otro tipo de servicios "espirituales" como lectura del Tarot, de la mano y "fotos del aura". El servicio de estos centros no parece estar ordenado por reglamentos específicos que certifiquen los servicios que prestan, más allá de los de salubridad o de operación por parte del municipio.

\section{El pueblo turista y el pueblo en resistencia}

Una de las características centrales de Tepoztlán es la intersubjetividad bajo la cual se construye la definición de este territorio; los habitantes, residentes foráneos, turistas y las autoridades (locales, estatales y nacionales) tiene visiones e intereses muy diferentes, (incluso encontrados) sobre el presente y futuro de este lugar.

Tepoztlán es al mismo tiempo un lugar con una población que guarda un sentido de comunidad "campesina", pueblo "mexicano", autodefinida como lugar de luchadores sociales, orgulloso por su resistencia contra proyectos (particularmente turísticos), valle sagrado, zona de turismo para los citadinos, cantina de fin de semana y zona de ovnis (Martin, 1990; Lomnitz-Adler, 1995; Scheinfeld, 1999; Velázquez-García, 2008; 2009 y 2012).

En este PM el turismo es la actividad económica de mayor crecimiento desde la década de los 80 en el siglo pasado. Esto ha generado un cambio en la intencionalidad de las autoridades locales y de una creciente parte de la población, aunque sin generar necesariamente un acuerdo sobre los planes que permitan definir el desarrollo para esta comunidad. En 1994 existían 201 sitios de hospedaje (6 mil 060 cuartos), 402 lugares de venta de comida y 448 mil 553 turistas habían ocupado un

2 El Temazcal es un baño de vapor prehispánico construido de piedra y lodo, utilizado con fines medicinales. 
cuarto. Acorde con el Anuario Estadístico del Estado de Morelos (INEGI, 2005; 2011), para el año 2009 existían 560 sitios de hospedaje ( 11 mil 797), mil 223 restaurantes y un millón 346 mil 394 turistas ocuparon un cuarto. Cabe mencionar que en el 2004 la cifra de los que se hospedaron llegó a los dos millones 198 mil 208.

La construcción por parte del Estado mexicano de Tepoztlán como un destino turístico por medio del PPM ha resultado en una mayor intersubjetividad entre los actores que están a favor y en contra del desarrollo de esta actividad. La mayoría de los habitantes están en una zona de grises donde aprovechan, pero al mismo tiempo critican, la actividad turística; algunos habitantes del pueblo han encontrado diversas formas para beneficiarse del continuo flujo de turistas acondicionando rudimentarias habitaciones que rentan, vendiendo cervezas, refrescos o utilizando sus patios como estacionamiento.

Por su parte, los trabajadores manuales como carpinteros, albañiles, plomeros y electricistas, construyen las casas u hoteles de los residentes nacionales o extranjeros. Los niveles de vida entre las personas que trabajan para el turismo en comparación con los de aquellos que viven de su trabajo en la tierra no son muy diferentes; no obstante, sus estilos de vida han experimentado cambios significativos, sobre todo en las aspiraciones educativas y la posición de las mujeres dentro de la familia (Martin, 1990).

En Tepoztlán el turismo no es una actividad aceptada por todos los residentes, por el contrario, han existido distintos tipos de resistencias y protestas, algunas abiertas y otras ocultas. En la plaza central del poblado se instala un tianguis que va contra la "imagen urbana" que pide el PPM; sin embargo, no ha logrado ser removido. Los pobladores y los vendedores consideran este lugar como importante para su vida cotidiana, sin importarles que los turistas lo vean "feo" o "sucio".

La presencia del tianguis (entre otras razones) le costó a Tepoztlán su salida del PPM en el 2009 (Velázquez-García, 2009a; Wahrhaftig, 2006). También existen mecanismos que buscan mantener el control del territorio en manos de los habitantes locales, la venta de propiedades a foráneos tiene que ser aprobaba por los vecinos originarios de la zona. Un recurso más evidente de resistencia es la exclusión de los recién llegados en las reuniones sobre problemas sociales o políticas públicas (Martin, 2005). Una forma más sutil de rechazo son los apodos que distinguen a los auténticos residentes de los extranjeros; los primeros son llamados tepoztecos, los segundos, "tepostizos"; es decir, habitantes verdaderos y falsos (Lomnitz-Adler, 1995). 
La intersubjetividad generada por la actividad turística, en el caso de Tepoztlán, no termina con actos aislados de repudio, sino que ha logrado estructurar protestas colectivas (Martin 1993; Lomnitz-Adler, 1995; Rosas, 1997; Reynolds, 1996 y 1997; Wahrhaftig, 2006; Velázquez-García, 2009a).

Los habitantes de Tepoztlán encabezaron un acto de rechazo contra los proyectos turísticos en 1960, cuando el presidente Adolfo López Mateos (1958-1964) presentó un plan para construir una carretera de vía rápida que conectara directamente a la Ciudad de México con Tepoztlán. Este proyecto, que buscaba incentivar el creciente flujo de turistas, fue rechazado por los pobladores. Los tepoztecos organizaron multitudinarias manifestaciones en la capital del estado, Cuernavaca, hasta que finalmente el proyecto fue cancelado (Velázquez-García, 2009a).

El proyecto turístico del presidente Adolfo López Mateos no fue el último. En 1962 la federación proyectó construir un complejo turístico, conformado por fraccionamientos residenciales con albercas y un club de golf. Años antes del inicio oficial del proyecto, corredores de bienes raíces habían adquirido ya 240 hectáreas de tierras dentro del Parque Nacional del Tepozteco (Sarmiento, 1997; Rosas, 1997). Esto generó un descontento generalizado de los habitantes de Tepoztlán, quienes organizaron marchas masivas en Cuernavaca (Scheinfeld, 1999; Velázquez-García, 2009a).

La autoridad recurrió a la cárcel y los arrestos extralegales y uno de los manifestantes fue asesinado presuntamente por policías, provocando gran indignación. Como resultado, el gobierno decidió cancelar el plan de manera definitiva (Centro de Derechos Humanos Miguel Agustín Pro, Cdhmap, 2004; Rosas, 1997).

En 1976 el gobierno federal proyectó construir un teleférico que correría desde la pirámide ubicada en el Cerro del Tepozteco hasta el centro del pueblo. Sin embargo, la idea nunca se concretó por falta de presupuesto (Scheinfeld, 1999). En 1986, el gobierno federal propuso construir una carretera que rodeara la localidad, pero tampoco contó con financiamiento. En 1991 fue propuesto nuevamente el tren escénico con fines turísticos, así como la apertura de establecimientos de franquicias comerciales. Todo ello fue sistemáticamente rechazado por la población local (Piqueras, 2000; Velázquez-García, 2009a).

Durante estos conflictos contra los proyectos, una mayoría de los habitantes de Tepoztlán continúa identificándose como campesinos, esto repercute en las zonas problemáticas y las que no lo son, es decir, en su calidad de campesinos determi- 
nadas actividades o necesidades del turismo (desarrollo de grandes hoteles, restaurantes lujosos que desplazaran a las personas del centro e incluso la idea de tener que dejar de tronar "cuetes" para no molestar a los visitantes, entre otras) son percibidas como contrarias a sus costumbres y prácticas.

El significado de esta identidad es una de las principales fuentes de pugna del pueblo con el Estado mexicano (Martin, 1993). Los tepoztecos han construido un discurso local de autenticidad utilizando algunas de las cualidades del campesino mitificado. Por su parte, el Estado mexicano posrevolucionario se autoproclamó portador de las promesas de la Revolución, por lo tanto, era el único que podía declarar una rebelión como legítima y posible (Martin, 1993).

Esto ha hecho que ambos grupos, tepoztecos y el Estado, se enfrenten respecto a los planes para el desarrollo local; los dos creían tener legitimidad para decidir sobre ello (Quero, 2002, Lomnitz-Adler, 1995). Los pobladores de Tepoztlán han percibido correctamente que el modelo de desarrollo turístico aplicado por el Estado mexicano significa una pérdida de la capacidad de decisión de las comunidades sobre el uso de sus espacios públicos y recursos; la realidad ordenada y objetivada de los planes de turismo que han buscado desarrollarse en esta población se encontró con una realidad difusa y más compleja de lo esperado.

La oposición más significativa en Tepoztlán contra un proyecto turístico de gran envergadura se produjo a finales de la década de los 90. En 1994, la empresa Kladt-Sobrino (KS), con el respaldo estatal, presentó un nuevo proyecto turístico para Tepoztlán. La obra estaba ubicada dentro del Parque Nacional del Tepozteco e incluía 187 hectáreas con casa club, campo de golf ( 18 hoyos), desarrollo residencial en condominio (800 lotes), centro deportivo de tenis (18 canchas), hotel (30 habitaciones), academia de golf y parque industrial corporativo (desarrollo de tecnología) (Martin, 1990; Rosas, 1997; Scheinfeld, 1999).

Desde el inicio de la construcción la obra estuvo repleta de irregularidades. Por ejemplo, no se realizó una consulta pública contemplada en la legislación ambiental mexicana vigente (Rosas, 1997; Velázquez-García, 2008 y 2009a; Weinberg, 1996). Esta movilización ambiental de Tepoztlán atrajo la atención nacional e internacional. Fue significativo el apoyo mostrado por organizaciones ecologistas internacionales como Corporate Accountability Research Group, Sierra Club, Greenpeace USA, Friends of the Earth, Global Trade Watch y People pro-Parks (Velázquez-García, 2005; 2008). 
Adicionalmente, la presencia del Ejército Zapatista de Liberación Nacional en Chiapas convertía en muy costosa la decisión de tomar militarmente el pueblo y resolver el conflicto mediante la represión. Cabe señalar que durante esta protesta se produjeron actos aislados de xenofobia contra los pobladores foráneos, aunque apoyaran al movimiento (Weinberg, 1996). El gobierno estatal giró órdenes de aprehensión contra los dirigentes. En abril de 1996 se produjo un enfrentamiento entre policías y tepoztecos, el resultado fue de 34 personas detenidas y varios heridos. Este hecho de violencia ocupó todos los espacios de noticias nacionales, tanto de radio como de televisión (Rosas, 1997).

Al día siguiente del enfrentamiento fue encontrado el cadáver de una de las personas que participó en la trifulca y que había sido arrestada por los policías. Diversos medios de información criticaron la actuación del gobierno estatal y el acontecimiento fue ampliamente difundido por las organizaciones internacionales que apoyaban al movimiento (Rosas, 1997; Cdhmap, 2004; Instituto Nacional de Ecología, 2004; Velázquez-García, 2005). El 12 de abril la principal compañía inversionista en este esquema anunció la cancelación del proyecto para construir el club de golf.

\section{Conclusiones}

El turismo es un fenómeno social que implica la interacción entre grupos sociales que guardan notables diferencias en los intereses, liderazgos, sentidos y valores que guían su acción. El análisis de las políticas públicas sobre turismo requiere abandonar el cómodo puerto de los modelos administrativos y de negocios que dan por supuesto la igualdad de motivaciones económicas, administrativas y culturales en los actores que están involucrados en el turismo, aun contra su voluntad.

El modelo de la construcción estatal de las políticas públicas permite "desatar" algunos de los supuestos implícitos en los modelos de diseño de políticas públicas, relacionados con los actores que participan (mostrando la diversidad de posturas, intereses y motivaciones para participar), los tipos de problemas (las intricadas relaciones entre problemas económicos, políticos y culturales que buscan ser aislados artificialmente en la formulación de acciones de gobierno) y soluciones de la acción de gobierno (la existencia de un cúmulo de formas de acción gubernamentales que están esperando un problema para ser aplicadas, independientemente de su idoneidad). 
En este artículo analizamos la construcción estatal del PPM en el municipio de Tepoztlán. Esto permitió identificar aquellos elementos de la historia, la cultura y las características del lugar que fueron retomados por el Estado para considerar a esta localidad como una "solución" en su búsqueda por generar alternativas de desarrollo en pequeñas localidades, en este caso a través del turismo.

Como sostuvo Claudio Lomnitz-Adler (1995), Tepoztlán es una comunidad que pasó de estar en la periferia de la historia nacional a ocupar por algunos momentos un lugar central. La importancia de Tepoztlán surgió de los conflictos sociales relacionados al intento mexicano de desarrollar proyectos turísticos sin contar con el consentimiento de la mayoría de la población local.

Este artículo proporciona elementos que explican esa oposición social como una grave falla en el diseño de las políticas turísticas. Como buscamos demostrar, la política pública turística, escoge y aísla elementos culturales, espaciales y sociales de las comunidades buscando generar un "paquete" que resulte atractivo. Este modelo de construcción estatal de las políticas resulta problemático, porque no es capaz de captar la complejidad de las cuestiones generando respuestas parciales y que solo favorecen a porciones muy específicas de la población.

La formulación de las políticas turísticas requiere de un regreso a la propuesta original de Laswell (1951) quien consideraba que esta ciencia es interdisciplinaria, por lo que requiere de áreas como la sociología, la ciencia política, la antropología, para producir un conocimiento que rebase los modelos financieros o económicos. En este sentido, el PPM es una política pública que requiere de un rediseño general, de tal manera que le permita conocer y atender a las distintas comunidades en sus circunstancias particulares, así como considerar la solución de los problemas locales una prioridad y no una consecuencia de su acción.

\section{Referencias}

Azteca América. (2011, 10 de septiembre). Seres extraterrestres en Tepoztlán [video]. YouTube. https://bit.ly/3bvzURk

Animal.mx. (2020, 10 de septiembre). Tres lugares de México famosos por supuestos "avistamientos ovnis". https://bit.ly/3u1DtpD 
Berger, P. y Luckmann, T. (1967). The Social construction of reality: a treatise in the sociology of Knowledge. Anchor Books.

Bourdieu, P. (2014). Sobre el estado. Cursos en el Collège de France (1989-1992). Anagrama.

Cardozo, M. I. (2006). La evaluación de políticas públicas y programas públicos. Miguel Ángel Porrúa.

Centro de Derechos Humanos Miguel Agustín Pro (Cdhmap). (2004, 25 de agosto). Tepoztlán: el derecho de un pueblo a sobrevivir. https://bit.ly/3u5oATh

Chase, S. (1931). Mexico: a study of two americas. Macmillan Company.

Balslev, H. (2008). Juntos pero no revueltos: Un estudio de caso sobre los migrantes norteamericanos en un pueblo mexicano; Transnacionalismo y asimilación. Copenhagen Business School. https://bit.ly/2O314pK

Cohen, M.D., March, J.G.y Olsen, J.P.(1972). Agarbage can model of organization choice. Administrative Science Quarterly, 17(1), 1-25. https://doi.org/10.2307/2392088

Concheiro, L. (1998). Tepoztlán un espacio en el tiempo un lugar en la historia. En P. Sánchez (Ed.), Antología histórica de Tepoztlán (pp. 277- 309). Instituto Nacional de Antropología e Historia.

Diario de Morelos. (2020, 10 de septiembre). Experimentan en Tepoztlán Morelos avistamiento de Ovni. https://bit.ly/3aoQXUY

Dean, M. (1999). Governmentality, power and rule in modern society. Sage publications.

Fog, J. y Kvale, S. (1992). Artikler om interviews. Aarhus, Dinamarca: Center for Kvalitativ Metodeudvikling. Psykologisk Institut y Aarhus Universitet.

Foucault, M. (1991). Governmentality. En B. Graham, C. Gordon y P. Miller (Eds.), The Foucault Effect: Studies in Governmentality (pp. 87- 104). Chicago University Press.

Foucault, M. (2004). Seguridad, territorio y población. Fondo de Cultura Económica.

González, J. (2012, 28 de septiembre). Turismo ufológico: lugares donde se pueden ver ovnis. Realidad OVNI. https://bit.ly/3b6ofaw

Graburn, N. (1989). Turismo el viaje sagrado. En V. Smith (Ed.), Anfitriones e invitados. Antropología del turismo (pp. 45- 68). Ediciones Endymion. 
Hiernaux, D. (1997). Los límites del turismo de masas. Revista Mexicana del Caribe, 4, 184-199. https://bit.ly/38pammZ

Instituto Nacional de Ecología (INE). (2004). Las decisiones y los días. https://bit.ly/ $3 \mathrm{bxaC5s}$

Instituto Nacional de Estadística y Geografía (INEGI). (2005). Anuario Estadístico del Estado de Morelos. https://bit.ly/3113EQe

Instituto Nacional de Estadística y Geografía (INEGI). (2011). Anuario Estadístico del Estado de Morelos. https://bit.ly/3dm8NYQ

Instituto Nacional de Estadística y Geografía (INEGI). (2015). Panorama sociodemográfico de Morelos 2015. https://bit.ly/3vbXQBI

Lara, O. (2004). Living and making social theory in Tepoztlán: a study of anthropologists in a mexican village..

Laswell, H. (1951). The policy orientation. En D. Lerner y H. Laswell (Eds.), The policy sciences (pp. 3-15). Standford University Press.

Lewis, O. (1951). Life in a mexican village: Tepoztlán restudied. University of Illinois Press.

Lewis, O. (1959). An anthropological approach of family studies. The American Journal of Sociology, 55(5), 468-475. https://doi.org/10.1086/220587

Lewis, O. (1968). Tepoztlán un pueblo de México, Joaquín Mortiz.

Lomnitz-Adler, C. (1995). Las salidas del laberinto. Joaquín Mortiz y Planeta.

March, J. G. y Olsen, J. (1989), Rediscovering institutions: the organizacional basis of politics. The Free Press.

Martin, J. (1990). Motherhood and power: the production of a women's culture of politics in a mexican community. American Ethnologist, 17(3), 470-490. https://bit.ly/3saRNua

Martin, J. (1993). Contesting authenticity: battles over the representation of history in Morelos, Mexico. Ethnohistory, 40(3), 438-465. https://bit.ly/3ay2p0z

Martin, J. (2005). Tepoztlán and the transformation of the mexican state: the politics of loose connections. University of Arizona Press.

MacCannell, D. (1973). Staged authenticity: arrangements of social space in tourist settings. American Sociological Review, 79(3), 589-603. https://bit.ly/2ZI9GVn 
Millán, H. y Cota, C. (2021) Pueblos Mágicos, pobreza y desigualdad. Dimensiones turísticas, 5(8), 63-86. https://doi.org/10.47557/WXYU8819

Oseguera, A. (2008). De ritos y antropólogos. Perspectivas teóricas sobre el ritual indígena en la antropología realizada en México. Cuicuilco, 15(42), 97-118. https://bit.ly/3drPD5m

Piqueras, A. (2000). Las identidades colectivas frente a los retos de la mundialización capitalista. Dos casos de estudio en México. Revista Mexicana de Ciencias Políticas y Sociales, 44(179). https://bit.ly/3sqXV1H

Quero, M. (2002). Una periferia que pudo ser centro: sociedad civil y gobernabilidad en Tepoztlán. En B. Levy (Ed.), Crisis y conflicto en el capitalismo latinoamericano (pp. 103- 106). Consejo Latinoamericano de Ciencias Sociales.

Redclift, M. (1986). Peasant movements and urbanisation in contemporary Morelos: to what do we owe our ignorance? Bulletin of Latin American Research 5(1), 95-100. https://doi.org/10.2307/3338785

Redfield, R. (1930). Tepoztlán, a mexican village: a study of folk life. University of Chicago Press.

Redfield, R. (1953). The primitive world and its transformations. Cornell University Press.

Redfield, R. (1955). The little community: viewpoints for the study of a human whole. University of Chicago Press.

Reynolds, J. (1996). La Guerra del Golf en el valle sagrado (primera parte). El Andar Magazine. https://bit.ly/3qt3ITK

Reynolds, J. (1997). La Guerra del Golf en el valle sagrado (segunda parte). El Andar Magazine. https://bit.ly/3rX9KfY

Rosas, M. (1997). Crónicas de desacatos y resistencias. Era.

Ruzo, D. (2012). El valle sagrado de Tepoztlán. Los templos Atlantes de México. Punto de Lectura.

Sánchez, R. (2005). La construcción social del poder local. El Colegio de México.

Sarmiento Silva, S. (1997). Morelos: sociedad, economía, política y cultura. Universidad Nacional Autónoma de México. 
Schneider A. e Ingram, H. (1993). Social construction of target population: implications for politics and policy. The American Political Review, 87(2), 334-347. https://doi.org/10.2307/2939044

Secretaría de Turismo (Sectur). (2019, 19 de septiembre). Pueblos Mágicos, Tepoztlán, Morelos. https://bit.ly/3u2VeVS

Secretaría de Turismo (Sectur). (2020). Programa Pueblos Mágicos. https://bit.ly/ 2OliAQk

Scheinfeld, E. (1999). Proyectos de inversión y conflictos ambientales. Instituto Nacional de Ecología.

Spradley, J. (1990). Participant observation. Harcourt Brace Jovanovich.

Urry, J. (2011). The tourist gaze. Sage

Velázquez-García, M. A. (2005). Relaciones entre organizaciones y movimientos sociales. Redes y oportunidades políticas: los casos de la Red Nacional de Acción Ecologista (Argentina) y la Red Nacional de Derecho a la información ambiental (México). Región y sociedad, 33, 33-70. https://bit.ly/3t5CXG8

Velázquez-García, M. A. (2008). La construcción de un movimiento ambiental en México: El club de golf en Tepoztlán, Morelos. Región y Sociedad, 20(43), 61-96. https://bit.ly/3bmbfxh

Velázquez-García, M. A. (2009a). Las luchas verdes: Los movimientos ambientalistas de Tepoztlán, Morelos y el Cytrar en Hermosillo, Sonora. Sonora, México. El Colegio de Sonora.

Velázquez-García, M. A. (2009b). Comunidad y utopías en los movimientos indígenas y campesinos en Latinoamérica. En H. Balslev, J. Gustafsson y M. A. Velázquez-García (Eds.), Utopías y globalización (pp. 51-76). El Colegio de Sonora.

Velázquez-García, M. A. y Balslev, H. (2012). Tepoztlán, una economía de la experiencia íntima. Latin American Research Review, 47(3), 134-154. https://doi. org/10.1353/lar.2012.0033

Velázquez-García, M. A. y Balslev, H. (2020). Los norteamericanos que reinventaron a los pueblos de México: Los emprendedores extranjeros en la redefinición de la cultura y el turismo. Latin American Research Review, 55(1), 49-63. http:// doi.org/10.25222/larr.110 
Velázquez García, M. A. y Lara Figueroa, H. N. (2017). El fomento y promoción del sector turístico en México. Estudios de caso y recomendaciones de política pública. El Colegio del Estado de Hidalgo.

Wahrhaftig, A. (2006, 15-18 de marzo). Pictorial state of the culture representations in Tepoztlán, Morelos [ponencia]. Reunión anual 2006. Simposio de la Latin American Studies Association, San Juan, Puerto Rico. 